\section{Colite ulcerativa inespecífica em crianças e adolescentes: análise de casuística}

\section{Non-specific ulcerative colitis in children and adolescents: casuistic analysis}

\author{
Adelma A. de Figueirêdo 1 \\ Joaquim A. C. Mota 2 \\ Francisco J. Penna 3 \\ Gisélia Alves Pontes Silva 4 \\ 1 Departamento clínico. Faculdade de Medicina. \\ Centro de Ciências Biológicas e da Saúde. \\ Universidade Federal de Roraima. Av. Ene Garcês, 2413. \\ Boa Vista, RR, Brasil. CEP: 69.304-000 \\ 2,3 Departamento de Pediatria. Faculdade de Medicina. \\ Universidade Federal de Minas Gerais. \\ Belo Horizonte, MG, Brasil \\ 4 Departamento de Pediatria. Faculdade de Medicina. \\ Universidade Federal de Pernambuco, Recife, PE, Brasil
}

\begin{abstract}
Objectives: to describe a series of non-specific ulcerative colitis with focus on the initial manifestation of the disease, propedeutics and interval between the first symptoms and diagnosis.

Methods: retrospective analysis of non-specific ulcerative colitis with symptoms appearing before 20 years old. Patients were selected in the Pediatrics Gastroenterology Services of the Hospital das Clinicas da Universidade Federal de Minas Gerais and of the Instituto Materno Infantil de Pernambuco.

Results: 29 patients with the diagnosis of nonspecific ulcerative colitis were identified with fifteen patients $(51.7 \%)$ of the male sex. The age of the beginning of the symptoms varied between 0.3 to 15.8. The most frequent symptoms of the diagnosis were diarrhea (89.7\%) and abdominal pain (82.8\%). The most common extra-intestinal manifestation was arthralgia/arthritis (41.4\%) The weight for age ratio was below the 3 percentile in $13.8 \%$ of the patients. Time interval between symptoms appearance and diagnosis varied between 01 and 10.9 years old.

Conclusions: time interval between initial symptoms and diagnosis remains high in our Region due to many different factors.
\end{abstract}

Key words Ulcerative colities, Adolescent, Child

\section{Resumo}

Objetivos: descrever uma série de casos de colite ulcerativa inespecífica, com ênfase nas manifestações iniciais, propedêtica e intervalo entre os primeiros sintomas e o diagnóstico.

Métodos: análise retrospectiva de casos de colite ulcerativa inespecífica, cujos sintomas iniciaram antes de 20 anos de idade. Os pacientes foram selecionados nos Serviços de Gastroenterologia Pediátrica do Hospital das Clínicas da Universidade Federal de Minas Gerais e do Instituto Materno Infantil de Pernambuco.

Resultados: foram encontrados 29 pacientes com diagnóstico de colite ulcerativa inespecífica, sendo $15(51,7 \%)$ pacientes do sexo masculino. A idade ao início dos sintomas variou entre 0,3 a 15,8 anos. Os sintomas mais freqüentes ao diagnóstico foram diarréia $(89,7 \%)$ e dor abdominal (82,8\%). A manifestação extra-intestinal mais comum foi artralgialartrite $(41,4 \%)$. A relação peso para a idade estava abaixo do percentil $3 \mathrm{em} \mathrm{13,8 \%} \mathrm{dos} \mathrm{pacientes.} O$ intervalo de tempo entre o início dos sintomas e o diagnóstico variou entre 0,1 e 10,9 anos.

Conclusões: o intervalo entre os sintomas iniciais e o diagnóstico ainda continua elevado na nossa Região devido ao somatório de vários fatores.

Palavras-chave Colite ulcerativa, Adolescente, Criança 


\section{Introdução}

Colite ulcerativa inespecífica (CUI) é um processo inflamatório crônico, restrito às camadas mucosa $\mathrm{e}$ submucosa do intestino grosso, que geralmente tem início no reto, podendo estender-se cranialmente, sempre em continuidade, comprometendo parte ou todo o cólon. Essa afecção, juntamente com a doença de Crohn, compõe as doenças inflamatórias intestinais. Em aproximadamente $10 \%$ dos casos não é possível, no momento do diagnóstico, a diferenciação entre a CUI e a doença de Crohn colônica. 1 Essas enfermidades vêm aumentando de incidência nas últimas décadas, especialmente a doença de Crohn, apesar de algumas casuísticas apontarem maior incremento da incidência da CUI que da doença de Crohn. ${ }^{2}$ A CUI foi identificada em crianças desde 1920, porém até hoje é pouco lembrada como afecção possível no grupo pediátrico. ${ }^{3}$ Aproximadamente $20 \%$ de todos os casos de CUI iniciam a sintomatologia antes dos 20 anos de idade. Nessa faixa etária, a doença incide mais em adolescentes que em crianças, embora possa acometer até lactentes. 1,4,5

O quadro clínico da CUI nos pacientes pediátricos é menos típico que em adultos. Muitas vezes, os sintomas intestinais são discretos ou inexistentes por meses ou anos, durante os quais predominam as manifestações extra-intestinais ou as repercussões sistêmicas da doença. Fato que torna o diagnóstico mais difícil nessa faixa etária.6-8 Essa dificuldade colide com a necessidade de diagnóstico mais precoce para esses pacientes que para adultos, devido as suas particularidades, como velocidade de crescimento elevada, mudanças físicas e labilidade psíquica, que os tornam mais susceptíveis às conseqüências do atraso no diagnóstico. ${ }^{8,9}$ Além disso, a CUI em crianças e adolescentes tem propensão para curso mais complicado, com tendência a extensão pancolônica e malignização ao longo da evolução. 10-14

No nosso país, a prevalência elevada das doenças infectoparasitárias, as quais podem manifestar-se com colite, é uma dificuldade a mais para o diagnóstico da CUI, sobretudo se há resistência em se pensar nessa afecção em crianças. Embora as afecções infectoparasitárias devam ser consideradas por sua importância epidemiológica, principalmente nas classes economicamente desfavorecidas, não podemos negar a esses pacientes a chance de diagnóstico diferencial com precocidade, quando for necessário.15,16

As manifestações iniciais e os métodos diagnósticos da CUI já estão bastante consolidados na literatura. Contudo, a pequena divulgação das casuísticas nacionais contribui para a não uniformização dos dados e a perpetuação de possíveis erros. O objetivo deste trabalho é descrever uma série de casos de CUI em crianças e adolescentes, com ênfase nas formas de apresentação, na propedêutica e no intervalo entre o primeiro sintoma e o diagnóstico.

\section{Métodos}

O estudo foi delineado como uma série de casos de CUI, cujos sintomas iniciaram antes de 20 anos de idade. Os pacientes foram selecionados através de busca ativa nos registros ambulatoriais dos Serviços de Gastroenterologia Pediátrica do Hospital das Clínicas (HC) da Universidade Federal de Minas Gerais (UFMG), e do Instituto Materno Infantil de Pernambuco (IMIP), Recife, Pernambuco, Brasil. Ambos são hospitais terciários que atendem aos usuários do Sistema Único de Saúde e estão localizados em grandes centros urbanos, das regiões Sudeste e Nordeste do país, respectivamente. Encontraram-se 29 pacientes com diagnóstico de CUI, atendidos entre outubro de 1987 e março de 2001. Foram considerados casos aqueles pacientes que preencheram um ou mais dos seguintes critérios: a) processo inflamatório restrito a mucosa e submucosa dos cólons, com depleção de mucina e abscessos crípticos; b) processo inflamatório inespecífico na mucosa e submucosa colônicas, sem presença de granulomas não caseosos; c) pancolite endoscópica e ileíte distal, essa sem ulcerações; d) alterações contínuas da mucosa colônica, vistas ao enema opaco e/ou endoscopicamente, em paciente com clínica sugestiva de CUI.

As informações relevantes para o estudo foram anotadas em protocolo confeccionado especificamente para permitir conhecer aspectos clínicos e diagnósticos. Nessa ferramenta constavam as seguintes variáveis: sexo, procedência, idade ao início dos sintomas, idade ao diagnóstico, manifestações clínicas (intestinais e extra-intestinais), dados antropométricos do National Center for Health Statistics (NCHS), história familiar e exames complementares realizados até o diagnóstico.

Esse estudo foi aprovado pelos Comitês de Ética em Pesquisa da UFMG e do IMIP.

\section{Resultados}

Quinze dos 29 (51,7\%) pacientes são do sexo masculino. Treze $(44,8 \%)$ pacientes residiam na capital ou região metropolitana, os demais eram procedentes do interior. A idade ao início dos sintomas variou entre 0,3 e 15,8 anos, com média e mediana de 6,6 e 5,7 anos, respectivamente. Apenas 7 $(24,1 \%)$ pacientes estavam acima de 10 anos de idade ao início do quadro clínico (Figura 1). 
Distribuição de 29 pacientes com colite ulcerativa inespecífica segundo a idade ao início dos sintomas e o intervalo de tempo entre os sintomas iniciais e o diagnóstico. Hospital das Clínicas da Universidade Federal de Minas Gerais e Instituto Materno Infantil de Pernambuco, IMIP, outubro de 1987 a março de 2001.

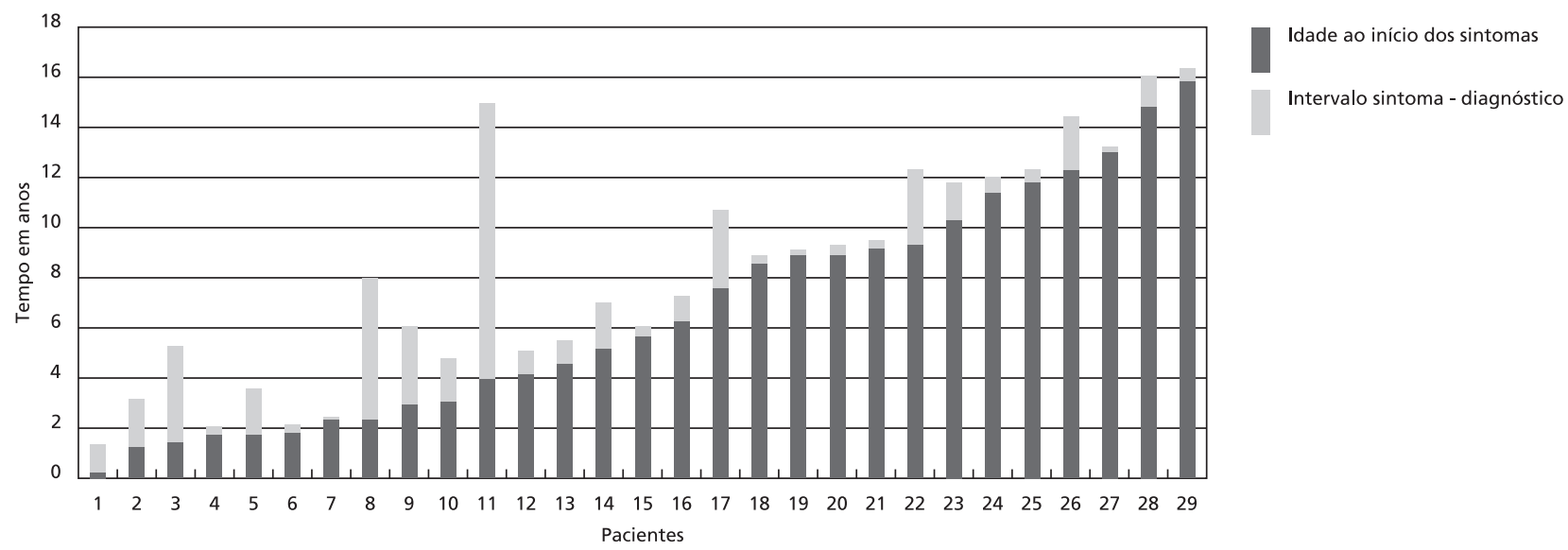

Os sintomas mais freqüentes ao diagnóstico foram diarréia $(89,7 \%)$ e dor abdominal (82,8\%) (Tabela 1$)$. A diarréia era intermitente na metade dos casos. A dor era referida difusamente por todo o abdômen em nove pacientes, pélvica em três, epigástrica e/ou em fossa ilíaca esquerda em outros três pacientes. Essa variável estava citada apenas em 15 prontuários. A manifestação extra-intestinal mais freqüente foi artralgia/artrite (12 pacientes), seguida por aftas de repetição (dois pacientes), pioderma gangrenoso (dois pacientes) e hepatopatias (dois pacientes). As manifestações osteoarticulares estavam em associação com outras manifestações extra-intestinais em três pacientes, dos quais dois tinham aftas de repetição e um hepatopatia. As artralgias/artrites antecederam os sintomas digestivos em cinco pacientes. Em dois desses, as queixas articulares surgiram vários anos antes do diagnóstico de CUI, sendo os pacientes submetidos à tratamentos reumatológicos. Um dos pacientes com doença hepática tinha colangite esclerosante primária e o outro não tinha diagnóstico definido da sua hepatopatia. A Tabela 2 discrimina os sintomas conforme o tipo das anotações nos prontuários.

A presença de anemia foi mensurada através da hematimetria em todos os pacientes. A hemoglobina média dos pacientes com anemia $(\mathrm{n}=16)$ foi igual a $9,0 \mathrm{~g} / \mathrm{dl}$. A relação peso para a idade estava abaixo do percentil 3 à primeira consulta em $13,8 \%(n=4)$ dos pacientes (Tabela 3).

\section{Tabela 1}

Sintomas iniciais da colite ulcerativa inespecífica em 29 crianças e adolescentes. Hospital das Clínicas da Universidade Federal de Minas Gerais e Instituto Materno Infantil de Pernambuco,IMIP, outubro de 1987 a março de 2001.

\begin{tabular}{lcc}
\hline Sintomas & N & $\%$ \\
\hline Diarréia & 26 & 89,7 \\
Dor abdominal & 24 & 82,8 \\
Diarréia com sangue & 22 & 75,9 \\
Perda de peso & 16 & 55,2 \\
Anemia & 16 & 55,2 \\
Manifestações extra-intestinais & 15 & 51,7 \\
Febre & 15 & 51,7 \\
Evacuação noturna & 12 & 41,4 \\
Anorexia / Hiporexia & 12 & 41,4 \\
Sangramento retal & 11 & 37,9 \\
Vômitos & 8 & 27,6 \\
Constipação & 6 & 20,7 \\
Inapetência & 6 & 20,7 \\
Urgência evacuatória & 6 & 20,7 \\
Tenesmo & 4 & 13,8 \\
\hline
\end{tabular}

Esta tabela representa o número vezes que cada sintoma foi positivo nos prontuários, mas não informa em quantos prontuários havia referência ao sintoma. 
Tabela 2

Discriminação dos sintomas iniciais da colite ulcerativa inespecífica em 29 crianças e adolescentes. Hospital das Clínicas da Universidade Federal de Minas Gerais e Instituto Materno Infantil de Pernambuco, IMIP, outubro de 1987 a março de 2001.

\begin{tabular}{lrrr}
\hline Sintoma & Positivo & Negativo & Sem informação \\
\hline Dor abdominal & 24 & 4 & 1 \\
Alívio da dor com a evacuação & 1 & 8 & 15 \\
Perda de peso & 16 & 11 & 2 \\
Evacuação noturna & 12 & 8 & 9 \\
Sangramento retal & 11 & 17 & 1 \\
Resto alimentar nas fezes & 4 & 1 & 24 \\
Tenesmo & 4 & 3 & 22 \\
Urgência evacuatória & 6 & 5 & 18 \\
Febre & 15 & 13 & 1 \\
Anorexia & 12 & 10 & 7 \\
Inapetência & 6 & 12 & 11 \\
Artrites / artralgias & 12 & 7 & 10 \\
Lesões de pele & 4 & 6 & 21 \\
Hepatopatias & 2 & 6 & \\
\hline Iitem sem informaçă & & \\
\end{tabular}

O item sem informação indica ausência de referência ao sintoma no prontuário do paciente, mas não exclui a possibilidade desse ter sido perguntado pelo médico durante a consulta.

Tabela 3

Distribuição de 29 pacientes com colite ulcerativa inespecífica conforme a relação peso/idade à primeira consulta com gastroenterologista pediátrico. Hospital das Clínicas da Universidade Federal de Minas Gerais e Instituto Materno Infantil de Pernambuco, IMIP, outubro de 1987 a março de 2001.

\begin{tabular}{|c|c|c|c|c|c|c|c|}
\hline Pacientes & $99,9-97$ & $97-66$ & $66-50$ & $50-16$ & $16-3$ & $3-0,1$ & $<0,1$ \\
\hline $\mathrm{n}$ & 1 & 0 & 4 & 13 & 7 & 3 & 1 \\
\hline$\%$ & 3,5 & 0,0 & 13,8 & 44,8 & 24,1 & 10,3 & 3,5 \\
\hline
\end{tabular}

O intervalo de tempo entre o início dos sintomas e o diagnóstico variou entre 0,1 e 10,9 anos, média = 1,7 anos e mediana $=1,0$ ano; sendo que, sete pacientes foram diagnosticados até seis meses do início dos sintomas, onze dos seis meses até um ano, seis de um ano até dois anos e sete após dois anos do início dos sintomas (Figura 1). Tanto os pacientes com os menores intervalos diagnósticos $(n=7)$ quanto aqueles com os maiores intervalos $(n=7)$ apresentavam diarréia com sangue ou sangramento retal sem diarréia, exceto um paciente. Esse queixa- va-se de dor em região pélvica, que o despertava do sono, evacuação noturna e urgência evacuatória.

Para o diagnóstico de CUI foram realizadas colonoscopias em 21 pacientes e retossigmoidoscopias mais enema baritado nos oito restantes. Em todos exames endoscópicos foram realizadas biópsias da mucosa intestinal para estudo histopatológico, porém não foram utilizados índices histológicos para o diagnóstico de CUI. A extensão do processo inflamatório estava restrita ao reto em dois pacientes, ao retossigmóide em quatro e em dois casos 
a inflamação progredia até o cólon esquerdo. Doze pacientes apresentavam pancolite e outros nove pancolite com ileíte de refluxo. A velocidade de hemossedimentação estava elevada $(>20 \mathrm{~mm} / \mathrm{h})$ em 16 dos $21(76,2 \%)$ pacientes que realizaram o exame. A proteína $\mathrm{C}$ reativa foi qualitativamente positiva em 10 dos $12(83,3 \%)$ pacientes aferidos. A albumina sérica estava abaixo dos valores de referência $(3,2$ mg\%) em 5 dos 8 (62,5\%) pacientes mensurados. Evidenciou-se plaquetose $\left(>350.000 \mathrm{plaq} / \mathrm{mm}^{3}\right) \mathrm{em}$ 7 de 10 (70\%) pacientes.

Em relação à história familiar, um paciente tinha a mãe e a avó materna acometidas por colite ulcerativa inespecífica; dois pacientes da casuística eram irmãos; um paciente tinha a irmã acometida por doença de Crohn; dois pacientes tinham parentes de primeiro grau com Diabetes Mellitus tipo I e outros dois antecedentes familiares de psoríase e polipose colônica familiar, isoladamente.

\section{Discussão}

A presteza no diagnóstico da CUI é de grande importância por tratar-se de doença crônica, sem tratamento clínico curativo e cuja demora na terapêutica pode levar a seqüelas graves, como baixa estatura, desnutrição, transtornos psicossociais e câncer intestinal.

Na presente casuística não houve diferença significativa de freqüência entre os sexos. Os sintomas tiveram início antes dos 10 anos de idade em 75,8\% dos pacientes, diferentemente do que é observado na maior parte das casuísticas cujas médias de idade ao início dos sintomas estão na segunda década de vida. 2,5,17 Um motivo do desvio para baixo dessa média pode ser um viés de seleção, pois, apesar destes ambulatórios atenderem adolescentes até 20 anos de idade, o ingresso de pacientes nesses serviços se dá majoritariamente em idade inferior a 14 anos.

A prevalência dos sintomas assemelhou-se ao descrito na literatura, sendo os principais: diarréia com sangue, dor abdominal, perda de peso e anemia. ${ }^{15} \mathrm{~A}$ dor abdominal era difusa na maioria dos pacientes, em discordância com a literatura, onde geralmente ela é referida em abdômen inferior. 15 Essa diferença pode ser devida à falta de localização da dor em nove prontuários e à faixa etária mais baixa da presente casuística, pois quanto mais jovem a criança maior a dificuldade de se caracterizar o sintoma.

A perda de peso estava presente como sintoma em $55,2 \%$ dos pacientes e a relação peso para a idade estava abaixo do percentil 3 em 13,8\% dos casos na primeira consulta. O que ressalta a importância da avaliação antropométrica pelo pediatra.

A presença de diarréia com sangue não foi estatisticamente mais freqüente no grupo com menor intervalo diagnóstico, provavelmente, por ocorrer em crises, intercalada com períodos de hábito intestinal normal. Essas diarréias são, erradamente, interpretadas como episódios recorrentes de gastrenterite infecciosa e os pacientes não recebem a investigação necessária para o diagnóstico diferencial.

A média de 1,7 anos (19 meses) para o intervalo de tempo entre o início dos sintomas e o diagnóstico foi mais elevada que a referida na literatura. Em serviços de saúde que atendem a populações economicamente menos privilegiadas, como os dois hospitais deste estudo, há maior dificuldade de controle periódico dos pacientes. O que pode explicar, em parte, o maior atraso no diagnóstico. No trabalho de Spivack et al., 15 realizado em Nova York com crianças portadoras de doença inflamatória intestinal sem e com seguro saúde, encontrou-se intervalo diagnóstico médio de 10,3 meses para as crianças sem seguro e 2,7 meses para aquelas seguradas. Ratificando que, mesmo em locais onde há maior acesso à assistência médica, os pacientes do serviço público têm maior atraso no diagnóstico. Vários fatores corroboram para isto, entre eles, a alternância de médicos. A existência de um protocolo sistematizado para o diagnóstico provavelmente minimizaria esse problema.

As artralgias/artrites foram as manifestações extra-intestinais mais freqüentes $(41,4 \%)$, semelhante à maioria das casuísticas. ${ }^{16,17}$ Nenhum dos pacientes apresentou eritema nodoso ou uveíte. Porém, uveíte não foi pesquisada na maioria dos pacientes. $\mathrm{Na}$ casuística de Passo et al. 18 de 44 crianças com CUI tinham artralgias $(31,8 \%)$ e quatro artrites $(9,1 \%)$. Os dois pacientes da presente casuística com artrite e que haviam recebido tratamento para doenças reumatológicas tinham a relação peso para a idade abaixo da média, o que deveria ter sido utilizado como sinal de alerta para a investigação de outras afecções. É comum que a anamnese meticulosa e retrospectiva ao diagnóstico de CUI encontre sintomas mínimos que passaram despercebidos durante anos. $19 \mathrm{O}$ que poderia ser evitado através da utilização protocolos para o diagnóstico, constando as repercussões sistêmicas e as manifestações extra-intestinais das doenças inflamatórias intestinais. A incidência de hepatopatias $(6,9 \%)$ foi semellhante à maioria das casuísticas, embora seja difícil avaliar se esta reflete a realidade, pois não houve critérios uniformes para a triagem, tais como dosagem de enzi- 
mas hepáticas e colangiografia endoscópica retrógada.

Quanto ao diagnóstico, o colonoscópio de fibra ótica e os procedimentos anestésicos seguros em crianças, tornaram a colonoscopia o exame de eleição para diagnosticar CUI. Ela permite, além da visibilização completa dos cólons e do íleo distal, a realização de biópsias seriadas em todos os segmentos. ${ }^{20-23}$ Uma maior suspeição do diagnóstico e indicações precisas otimizariam a realização desse exame em serviços com recursos parcos. O enema opaco não tem a mesma sensibilidade que a colonoscopia com exame histológico. 23 A não realização desse último em todos os pacientes diminui a força do diagnóstico e prejudica a avaliação da extensão do processo inflamatório. Devido a esse fato não foram feitas inferências na casuística do presente estudo entre sintomas, atraso diagnóstico e extensão do processo inflamatório.

Avaliando-se separadamente cada prova inflamatória, elas foram positivas acima de $50 \%$ das vezes em que foram realizadas. À semelhança da importância de um protocolo para a anamnese, o estudo de Beattie et al.24 ilustra a importância da sistematização laboratorial ao investigar-se os casos suspeitos de CUI. De 13 crianças com a enfermidade apenas uma teve a triagem laboratorial (plaquetas, hemoglobina, velocidade de hemossedimentação, proteína $\mathrm{C}$ reativa e albumina) totalmente negativa, a despeito de sangramento retal evidente.

\section{Conclusões}

O intervalo diagnóstico ainda continua elevado no nosso meio, provavelmente pelo somatório de vários fatores, tais como, problemas socioeconômicos, dificuldades organizacionais e a falta de sistematização para o diagnóstico. Vimos nesta casuística que algumas variáveis importantes na caracterização da CUI não constavam nos prontuários. A utilização sistemática de um protocolo para o diagnóstico evitaria a perda de informações importantes, uniformizaria a investigação, viabilizaria a realização de estudos retrospectivos e, provavelmente, anteciparia o diagnóstico.

\section{Referências}

1. Baldassano RN, Piccoli DA. Inflammatory bowel disease in pediatric and adolescent patients. Gastroenterol Clin North Am 1999; 28: 445-58.

2. Lindendberg E, Lindquist B, Holmquist L, Hildebrand H. Inflammatory bowel disease in children and adolescents in Sweden,1984-1995. J Pediatr Gastroenterol Nutr 2000; 30: 259-64.

3. Walker-Smith JA. Chronic inflammatory bowel disease in children: a complex problem in management. Postgrad Med J 2000; 76: 469-72.

4. Russel MG. Changes in the incidence of inflammatory bowel disease: What does it mean? Eur J Intern Med 2000; 11: 191-6.

5. Falcone RA, Lewis LG, Warner BW. Predicting the need for colectomy in pediatric patients with ulcerative colitis. J Gastroenterol Surg 2000; 4: 201-6.

6. Sakata T, Niwa Y, Goto H, Hirooka Y, Hayakawa T, Ohmiya N, Kobayashi S. Asymptomatic inflammatory bowel disease with special reference to ulcerative colitis in apparently healthy persons. Am J Gastroenterol 2001; 96: 735-9.

7. Haller C, Markowitz JA. Perspective on inflammatory bowel disease in the child and adolescent at the turn of the millenium. Cur Gastroenterol Rep 2001; 3: 263-71.

8. Büller HA. Problems in diagnosis of IBD in children. Neth J Med 1997; 50: S8-S11.
9. Saha MT, Ruuska T, Laippala P, Lenko HI. Growth of pubertal children with inflammatory bowel disease. J Pediatr Gastroenterol Nutr 1998; 26: 310-4.

10. Rivosecchi M, Lucchetti MC, Dall'Oglio L, Ponticelli A, Federici G, Zaccara A, Ferretti F, Castro M. Ulcerative colitis in children under 10 years of age: medical and surgical treatment. Acta Chir Belg 1996; 96: 104-7.

11. Verhave M, Winter HS, Grand RJ. Azathioprine in the treatment of children with inflammatory bowel disease. J Pediatr 1990; 117: 809-14

12. Ferguson A. Assessment and management of ulcerative colitis in children. Eur Gastroenterol Hepatol 1997; 9: 958-63.

13. Mir-Madjlessi SH, Michener WM, Farmer RG. Course and prognosis of Idiopathic ulcerative proctosigmoiditis in young patients. J Pediatr Gastroenterol Nutr 1986; 5: 571-5.

14. Kirschner BS. Ulcerative colitis in children. Pediatr Clin N Am 1996; 43: 235-54

15. Spivak W, Sockolow R, Rigas A. The relationship between insurance class and severity of presentation of inflammatory bowel disease in children. Am J Gastroenterol 1995; 90: 982-7.

16. Watson AJ, Johnston AT, Barker PM, Youngson GG, Bisset WM, Mahomed AA. The presentation and management of juvenile-onset chronic inflammatory bowel disease in 
Northeastern Scotland. J Pediatr Surg 2002; 37: 83-6.

17. Hyams JS. Extraintestinal manifestations of inflammatory bowel disease in children. J Pediatr Gastroenterol Nutr 1994; 19: 7-21

18. Passo MH, Fitzgerald JF, Brandt KD. Arthritis associated with inflammatory bowel disease in children. Relationship of joint disease to activity and severity of bowel lesion. Dig Dis Sci 1986; 31: 492-7.

19. Israel DM, Olson AD, Ilowite NT, Davidson M. Arthritis as the initial manifestation of inflammatory bowel disease in early infancy. J Pediatr Gastroenterol Nutr 1989; 9 123-5.
20. Goldman H. Colonic mucosal biopsy in inflammatory bowel disease. Surg Pathol 1991; 4: 3-24.

22. Chong SKF, Blackshaw AJ, Boyle S, Williams CB, WalkerSmith JA. Histological diagnosis of chronic inflammatory bowel disease in childhood. Gut 1985; 26: 55-9.

23. Aideyan UO, Smith WL. Inflammatory bowel disease in children. Radiol Clin N Am 1996; 34: 885-901.

24. Beattie RM, Walker-Smith JA, Murch SH. Indications for investigation of chronic gastrointestinal symptoms. Arch Dis Child 1995; 73: 354-5.

Recebido em 26 de janeiro de 2004.

Versão final apresentada em 24 de maio de 2004

Aprovado em 21 de julho de 2004 